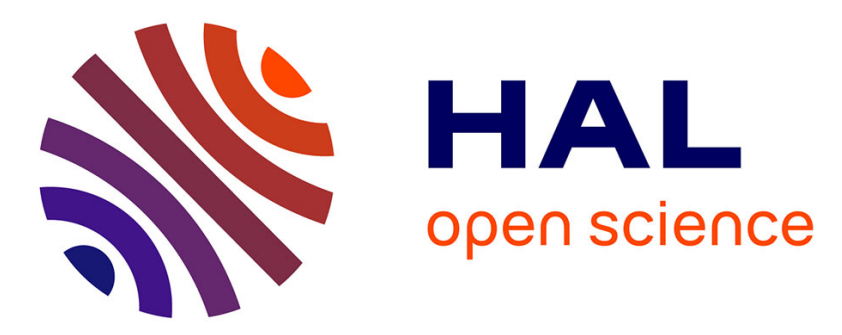

\title{
A fast algorithm for the computation of 2-D forward and inverse MDCT
}

\author{
Jiasong Wu, Huazhong Shu, Lotfi Senhadji, Limin M. Luo
}

\section{To cite this version:}

Jiasong Wu, Huazhong Shu, Lotfi Senhadji, Limin M. Luo. A fast algorithm for the computation of 2-D forward and inverse MDCT. Signal Processing, 2008, 88 (6), pp.1436-1446. 10.1016/j.sigpro.2007.12.003 . hal-00271377

\section{HAL Id: hal-00271377 \\ https://hal.science/hal-00271377}

Submitted on 22 Jul 2008

HAL is a multi-disciplinary open access archive for the deposit and dissemination of scientific research documents, whether they are published or not. The documents may come from teaching and research institutions in France or abroad, or from public or private research centers.
L'archive ouverte pluridisciplinaire HAL, est destinée au dépôt et à la diffusion de documents scientifiques de niveau recherche, publiés ou non, émanant des établissements d'enseignement et de recherche français ou étrangers, des laboratoires publics ou privés. 


\title{
A fast algorithm for the computation of 2-D forward and inverse MDCT
}

$$
\text { J.S. Wu }{ }^{\text {a, d }} \text {, H.Z. Shu }{ }^{\text {a, d }} \text {, L. Senhadji }{ }^{\text {b, c, d }} \text {, L.M. Luo }{ }^{\text {a,d }}
$$

${ }^{a}$ Laboratory of Image Science and Technology, School of Computer Science and Engineering, Southeast University, 210096, Nanjing, China

${ }^{\mathrm{b}}$ INSERM, U642, Rennes, F-35000, France

'Université de Rennes 1, LTSI, Rennes, F-35000, France

${ }^{\mathrm{d}}$ Centre de Recherche en Information Biomédicale Sino-Français (CRIBs)

Information about the corresponding author:

Huazhong Shu, Ph.D

Laboratory of Image Science and Technology

School of Computer Science and Engineering

Southeast University, 210096, Nanjing, China

Tel: 00-86-25-83 794249

Fax: 00-86-25-83 792698

Email: shu.list@seu.edu.cn

\begin{abstract}
A fast algorithm for computing the two-dimensional (2-D) forward and inverse modified discrete cosine transform (MDCT and IMDCT) is proposed. The algorithm converts the 2-D MDCT and IMDCT with block size $M \times N$ into four 2-D discrete cosine transforms (DCTs) with block size $(M / 4) \times(N / 4)$. It is based on an algorithm recently presented by Cho [7] for the efficient calculation of one-dimensional MDCT and IMDCT. Comparison of the computational complexity with the traditional row-column method shows that the proposed algorithm reduces significantly the number of arithmetic operations.
\end{abstract}

Keywords - 2-D MDCT, 2-D DCT, fast algorithm, image coding 


\section{Introduction}

The forward and inverse modified discrete cosine transform (MDCT and IMDCT) are extensively used to realize the analysis/synthesis filter banks of time domain aliasing cancellation scheme for subband coding [1]. Such a filter bank is equivalent to the modulated lapped transform (MLT) introduced by Malvar [2]. Many fast algorithms have been reported in the literature for computing the one-dimensional (1-D) MDCT/IMDCT (or MLT/MLT ${ }^{-1}$ ). For example, Britanak and Rao [3] proposed an efficient approach for implementing the $M$-point MDCT and IMDCT based on the $M / 4$-point DCT/DST and corresponding $M / 4$-point IDCT/IDST, respectively. Lee [4] then suggested an improvement in the computational speed of this algorithm. By using a matrix representation, Cheng and Hsu [5] presented various approaches for efficient implementation of the MDCT and IMDCT. Recently, Truong et al. [6] developed a fast algorithm for computing the $M$-point MDCT and IMDCT through $M / 2$-point DCT. Among these approaches, the algorithms reported in [4], [5] and [6] are probably the most efficient for computing the MDCT in terms of the arithmetic complexity. However, the algorithm presented by Cho et al in [7], which does not contain recursive structure, seems to achieve a good balance between the arithmetic complexity and computational structure. A comprehensive list of references on this subject is available in [8] and [9]. The two-dimensional (2-D) MDCT/IMDCT (or MLT/MLT ${ }^{-1}$ ), belonging to the lapped transforms, have a better performance compared to the non-lapped transforms (like the 2-D DCT/IDCT), not only because they have higher coding gains, but also they lead to a strong reduction in "blocking effects" in image coding [10]. Therefore, the 2-D MDCT/IMDCT have found their applications in image coding $[11,12]$, spectral image analysis [13] and digital image 
watermarking [14].

During the past decades, many fast algorithms for computing the 1-D and 2-D DCT have been proposed [15-43]. A comprehensive survey of DCT algorithms can be found in [44] and comments on various fast algorithms for 2-D DCT was given in [45]. For the 1-D case, Lee's algorithm [15] and Hou's algorithm [16] are probably the most attractive radix-2 algorithms for computing the $2^{m}$-point DCT. Loeffler et al. [17] presented a fast algorithm for computing the 8- and 16-point DCT with minimum computational complexity. Chan and Siu [18] presented a mixed radix-3/6 algorithm to realize the DCT of length- $M=2^{m} 3^{n}, m, n \geq 1$. Kok [19] then suggested a generalized radix-2 algorithm that can be used to compute the even-length DCT. Recently, Bi and Yu [20] derived an efficient mixed-radix algorithm for computing the DCT of composite sequence length $M=p \cdot 2^{m}$ where $p$ is an odd integer.

For the 2-D case, fast DCT algorithms can be classified into three categories: indirect algorithms, direct algorithms and optimal algorithms based on complexity theory or tensor approach. The indirect algorithms calculate the 2-D DCT through other transforms such as 2-D fast Fourier transform (FFT) [21, 22], 4-D FFT [23], or polynomial transform [24-26]. Among them, by using a polynomial transform (PT), Duhamel and Guillemot [24] developed the most efficient 2-D DCT algorithm for the block sizes $2^{m} \times 2^{m}, m \geq 3$. Zeng et al. [26] also presented a PT-based multidimensional DCT algorithm, which can be used to compute the 2-D DCT for the rectangular block sizes $2^{m} \times 2^{n}, m, n \geq 2$. Tatsaki et al. [23] derived a prime-factor DCT algorithm for computing the 2-D DCT of the block size $N \times N$ with $N \neq 2^{m}$. The direct algorithms include the calculation of 2-D DCT through $N$ sets of $N$-point 1-D DCTs plus a post-addition stage [27-31], matrix factorization or recursive computation [32-37], 
constant geometry algorithm $[38,39]$, and Chebyshev polynomial [40]. Among them, Britanak and Rao [36] developed an efficient recursive 2-D DCT algorithm for a rectangular $2^{m} \times 2^{n}$ block sizes. Bi et al. [37] suggested an algorithm that supports transform sizes $p \cdot 2^{m} \times q \cdot 2^{n}$, where $p$ and $q$ are odd integers. Note that the algorithms reported in [28]-[30] and [35] require the same number of multiplications and similar number of additions as that of the algorithm presented in [24] for computing the $2^{m} \times 2^{m}$-point DCT, but they have more regular computational structures compared to [24]. The optimal algorithms based on complexity theory or tensor approach [41-43] are mainly proposed to reach the minimum multiplicative complexity. For example, by using the 1-D DCT-based tensor approach, Feig and Winograd [41] obtained the lower bound of the multiplicative complexity which is $2^{m}\left(2^{m+1}-m-2\right)$ for the $2^{m} \times 2^{m}$ block sizes DCT. That is to say, the lower bounds of the multiplicative complexity for $8 \times 8$ - and 16×16-point DCT are 88 and 416, respectively. As noted in [45], by combining Loteffler's 1-D DCT algorithm [17] with Cho's 2-D DCT algorithm [28], the multiplications needed for the $8 \times 8$ - and 16×16-point DCT are 88 and 496, respectively. Recently, by using the shifted Fourier transform-based tensor approach, Grigoryan and Agaian [43] proposed an approach in which 84 and 460 multiplications are required for computing the $8 \times 8$ - and 16×16-point DCTs, respectively. By utilizing the distributed arithmetic (DA) structure of the 2-D DCT, Pan [46] reported that only 64 multiplications are required for the computation of $8 \times 8$-point DCT. Although many algorithms have been reported to reduce the arithmetic complexity of 2-D DCT, to the authors' knowledge, little attention has been paid on the fast computation of 2-D MDCT/IMDCT. Frantzeskakis and Karathanasis [47] developed a time-recursive approach for real-time computation of the 2-D MLT. In most cases, the 2-D 
MDCT/IMDCT are calculated with the row-column method [11-14], which requires evaluating $M$ sets of $N$-point MDCTs/IMDCTs and $N$ sets of $M$-point MDCTs/IMDCTs for an $M \times N$-point 2-D MDCT/IMDCT. As noted in [24], the true 2-D techniques are more efficient than the row-column approach. Therefore, proper 2-D algorithms need to be developed.

In this paper, the 1-D MDCT/IMDCT algorithm presented in [7] is extended to two dimensions to obtain a new 2-D MDCT/IMDCT algorithm. In section II, a simple variation of the algorithm in [7] is described. The algorithm is then generalized to 2-D in Section III. The computational complexity of the method is analyzed and compared to the row-column method in Section IV. Section V concludes the work.

\section{1-D MDCT/IMDCT algorithm}

Let $\{x(m)\}, m \in[0, M-1]$ denote a windowed input data sequence. The unnormalized 1-D forward and inverse MDCT are respectively defined as [1]

$$
\begin{gathered}
X(k)=\sum_{m=0}^{M-1} x(m) \cos \left[\frac{\pi}{2 M}\left(2 m+1+\frac{M}{2}\right)(2 k+1)\right], k \in[0, M / 2-1], \\
\hat{x}(m)=\sum_{k=0}^{M / 2-1} X(k) \cos \left[\frac{\pi}{2 M}\left(2 m+1+\frac{M}{2}\right)(2 k+1)\right], m \in[0, M-1],
\end{gathered}
$$

where $M$ is assumed to be divisible by 4 , i.e., $M=4 p$.

In this section, we briefly describe the algorithm proposed in [7]. Using the following permutations introduced in [4] and [7]

$$
w(m)= \begin{cases}-x(3 M / 4-1-m)-x(3 M / 4+m), & m \in[0, M / 4-1] \\ x(m-M / 4)-x(3 M / 4-1-m), & m \in[M / 4, M / 2-1],\end{cases}
$$

and 


$$
y(m)= \begin{cases}-\hat{x}(3 M / 4+m), & m \in[0, M / 4-1] \\ \hat{x}(m-M / 4), & m \in[M / 4, M-1] .\end{cases}
$$

Equations (1) and (2) can be rewritten as

$$
\begin{aligned}
& X(k)=\sum_{m=0}^{M / 2-1} w(m) \cos \left[\frac{\pi}{2 M}(2 m+1)(2 k+1)\right], k \in[0, M / 2-1], \\
& y(m)=\sum_{k=0}^{M / 2-1} X(k) \cos \left[\frac{\pi}{2 M}(2 m+1)(2 k+1)\right], m \in[0, M / 2-1] .
\end{aligned}
$$

The above equations show that the forward and inverse MDCT can be realized by the same DCT-IV algorithm.

Equation (5) can further be computed as follows.

$$
\begin{aligned}
& A(k)=X(2 k)+X(2 k-1)=2 \sum_{m=0}^{M / 4-1} u(m) \cos \left[\frac{2 \pi}{M}(2 m+1) k\right], k \in[0, M / 4-1], \\
& B(k)=X(2 k)-X(2 k-1)=2 \sum_{m=0}^{M / 4-1} v(m) \sin \left[\frac{2 \pi}{M}(2 m+1) k\right], k \in[1, M / 4],
\end{aligned}
$$

where

$$
\begin{aligned}
& u(m)=w(m) \cos \left[\frac{\pi}{2 M}(2 m+1)\right]+w(M / 2-1-m) \sin \left[\frac{\pi}{2 M}(2 m+1)\right], m \in[0, M / 4-1], \\
& v(m)=-w(m) \sin \left[\frac{\pi}{2 M}(2 m+1)\right]+w(M / 2-1-m) \cos \left[\frac{\pi}{2 M}(2 m+1)\right], m \in[0, M / 4-1],
\end{aligned}
$$

with the initial conditions $X(0)=A(0)$ and $X(M / 2-1)=-B(M / 4)$.

\section{2-D MDCT/IMDCT algorithm}

The corresponding 2-D MDCT and IMDCT are respectively defined by

$$
\begin{gathered}
X(k, l)=\sum_{m=0}^{M-1} \sum_{n=0}^{N-1} x(m, n) \cos \left[\frac{\pi}{2 M}\left(2 m+1+\frac{M}{2}\right)(2 k+1)\right] \cos \left[\frac{\pi}{2 N}\left(2 n+1+\frac{N}{2}\right)(2 l+1)\right], \\
k \in[0, M / 2-1], l \in[0, N / 2-1]
\end{gathered}
$$




$$
\begin{gathered}
\hat{x}(m, n)=\sum_{k=0}^{M / 2-1} \sum_{l=0}^{N / 2-1} X(k, l) \cos \left[\frac{\pi}{2 M}\left(2 m+1+\frac{M}{2}\right)(2 k+1)\right] \cos \left[\frac{\pi}{2 N}\left(2 n+1+\frac{N}{2}\right)(2 l+1)\right], \\
m \in[0, M-1], n \in[0, N-1],
\end{gathered}
$$

where $M$ and $N$ are both assumed to be divisible by 4 .

Step 1: Mapping M $\times N$-point forward and inverse MDCT to $(M / 2) \times(N / 2)-$ point DCT-IV.

Letting

$w(m, n)$

$$
\begin{aligned}
& \int\left[x\left(\frac{3 M}{4}-1-m, \frac{3 N}{4}-1-n\right)+x\left(\frac{3 M}{4}-1-m, \frac{3 N}{4}+n\right)\right]+\left[x\left(\frac{3 M}{4}+m, \frac{3 N}{4}-1-n\right)\right. \\
& \left.+x\left(\frac{3 M}{4}+m, \frac{3 N}{4}+n\right)\right], \quad m \in\left[0, \frac{M}{4}-1\right], n \in\left[0, \frac{N}{4}-1\right] \\
& {\left[x\left(\frac{3 M}{4}-1-m, \frac{3 N}{4}-1-n\right)-x\left(\frac{3 M}{4}-1-m, n-\frac{N}{4}\right)\right]+\left[x\left(\frac{3 M}{4}+m, \frac{3 N}{4}-1-n\right)\right.} \\
& \left.-x\left(\frac{3 M}{4}+m, n-\frac{N}{4}\right)\right], \quad m \in\left[0, \frac{M}{4}-1\right], n \in\left[\frac{N}{4}, \frac{N}{2}-1\right] \\
& =\left\{\left[x\left(\frac{3 M}{4}-1-m, \frac{3 N}{4}-1-n\right)+x\left(\frac{3 M}{4}-1-m, \frac{3 N}{4}+n\right)\right]-\left[x\left(m-\frac{M}{4}, \frac{3 N}{4}-1-n\right)\right.\right. \\
& \left.+x\left(m-\frac{M}{4}, \frac{3 N}{4}+n\right)\right], \quad m \in\left[\frac{M}{4}, \frac{M}{2}-1\right], n \in\left[0, \frac{N}{4}-1\right] \\
& {\left[x\left(\frac{3 M}{4}-1-m, \frac{3 N}{4}-1-n\right)-x\left(\frac{3 M}{4}-1-m, n-\frac{N}{4}\right)\right]-\left[x\left(m-\frac{M}{4}, \frac{3}{4} N-1-n\right)\right.} \\
& \left.-x\left(m-\frac{M}{4}, n-\frac{N}{4}\right)\right], \quad m \in\left[\frac{M}{4}, \frac{M}{2}-1\right], n \in\left[\frac{N}{4}, \frac{N}{2}-1\right] \text {, }
\end{aligned}
$$

Eq. (11) becomes

$$
\begin{array}{r}
X(k, l)=\sum_{m=0}^{M / 2-1} \sum_{n=0}^{N / 2-1} w(m, n) \cos \left[\frac{\pi}{2 M}(2 m+1)(2 k+1)\right] \cos \left[\frac{\pi}{2 N}(2 n+1)(2 l+1)\right], \\
k \in[0, M / 2-1], l \in[0, N / 2-1] .
\end{array}
$$

Using a mapping analogous to (4)

$$
y(m, n)= \begin{cases}\hat{x}(3 M / 4+m, 3 N / 4+n), & m \in[0, M / 4-1], n \in[0, N / 4-1] \\ -\hat{x}(3 M / 4+m, n-N / 4), & m \in[0, M / 4-1], n \in[N / 4, N-1] \\ -\hat{x}(m-M / 4,3 N / 4+n), & m \in[M / 4, M-1], n \in[0, N / 4-1] \\ \hat{x}(m-M / 4, n-N / 4), & m \in[M / 4, M-1], n \in[N / 4, N-1],\end{cases}
$$


Equation (12) can be written as

$$
\begin{gathered}
y(m, n)=\sum_{k=0}^{M / 2-1} \sum_{l=0}^{N / 2-1} X(k, l) \cos \left[\frac{\pi}{2 M}(2 m+1)(2 k+1)\right] \cos \left[\frac{\pi}{2 N}(2 n+1)(2 l+1)\right], \\
m \in[0, M-1], n \in[0, N-1] .
\end{gathered}
$$

Note that

$$
\begin{array}{r}
y(m, n)=-y(M-1-m, n)=-y(m, N-1-n)=y(M-1-m, N-1-n), \\
m \in[0, M / 2-1], n \in[0, N / 2-1] .
\end{array}
$$

Therefore, only $y(m, n), m \in[0, M / 2-1], n \in[0, N / 2-1]$ needs to calculate. Equations (14) and (16) show that the 2-D forward and inverse MDCT can be realized by the same 2-D DCT-IV algorithm.

Step 2: Decomposing $(M / 2) \times(N / 2)-$ point DCT-IV into four $(M / 4) \times(N / 4)-p o i n t ~ D C T$.

Instead of computing (14) directly, we propose in this subsection an algorithm suitable for fast computation.

\section{Letting}

$$
\begin{aligned}
& C(k, l)=C_{1}(k, l)+C_{2}(k, l), \\
& D(k, l)=C_{1}(k, l)-C_{2}(k, l), \\
& C^{\prime}(k, l)=C_{1}^{\prime}(k, l)+C_{2}^{\prime}(k, l), \\
& D^{\prime}(k, l)=C_{1}^{\prime}(k, l)-C_{2}^{\prime}(k, l),
\end{aligned}
$$

where

$$
\begin{array}{ll}
C_{1}(k, l)=\frac{1}{4}[X(2 k, 2 l)+X(2 k, 2 l-1)], & k \in[0, M / 2-1], l \in[0, N / 2], \\
C_{2}(k, l)=\frac{1}{4}[X(2 k-1,2 l)+X(2 k-1,2 l-1)], & k \in[1, M / 2], l \in[0, N / 2], \\
C_{1}^{\prime}(k, l)=\frac{1}{4}[X(2 k, 2 l)-X(2 k, 2 l-1)], & k \in[0, M / 2-1], l \in[0, N / 2], \\
C_{2}^{\prime}(k, l)=\frac{1}{4}[X(2 k-1,2 l)-X(2 k-1,2 l-1)], & k \in[1, M / 2], l \in[0, N / 2] .
\end{array}
$$

\section{A. Computation of $C(k, l)$ and $D(k, l)$.}


1) Computation of $C_{1}(k, l)$ and $C_{2}(k, l)$.

From (22), we have

$$
\begin{aligned}
C_{1}(k, l)= & \frac{1}{4} \sum_{m=0}^{M / 2-1} \cos \left[\frac{\pi}{2 M}(2 m+1)(4 k+1)\right] \sum_{n=0}^{N / 2-1} w(m, n)\left\{\cos \left[\frac{\pi}{2 N}(2 n+1)(4 l+1)\right]\right. \\
& \left.+\cos \left[\frac{\pi}{2 N}(2 n+1)(4 l-1)\right]\right\} .
\end{aligned}
$$

Using (7), we can easily get

$$
C_{1}(k, l)=\frac{1}{2} \sum_{n=0}^{N / 4-1} \cos \left[\frac{2 \pi}{N}(2 n+1) l\right] \sum_{m=0}^{M / 2-1} u(m, n) \cos \left[\frac{\pi}{2 M}(2 m+1)(4 k+1)\right],
$$

where

$$
u(m, n)=w(m, n) \cos \left[\frac{\pi}{2 N}(2 n+1)\right]+w(m, N / 2-1-n) \sin \left[\frac{\pi}{2 N}(2 n+1)\right] .
$$

Similarly, we have

$$
C_{2}(k, l)=\frac{1}{2} \sum_{n=0}^{N / 4-1} \cos \left[\frac{2 \pi}{N}(2 n+1) l\right] \sum_{m=0}^{M / 2-1} u(m, n) \cos \left[\frac{\pi}{2 M}(2 m+1)(4 k-1)\right] .
$$

2) Computation of $C(k, l)$ and $D(k, l)$.

Substituting (27) and (29) into (18), we obtain

$$
\begin{gathered}
C(k, l)=\sum_{m=0}^{M / 4-1} \sum_{n=0}^{N / 4-1} u_{u}(m, n) \cos \left[\frac{2 \pi}{M}(2 m+1) k\right] \cos \left[\frac{2 \pi}{N}(2 n+1)\right], \\
k \in[0, M / 4-1], l \in[0, N / 4-1],
\end{gathered}
$$

where

$$
u_{u}(m, n)=u(m, n) \cos \left[\frac{\pi}{2 M}(2 m+1)\right]+u(M / 2-1-m, n) \sin \left[\frac{\pi}{2 M}(2 m+1)\right]
$$

For the computation of $D(k, l)$, we have

$$
\begin{aligned}
D(k, l)=\frac{1}{2} & \sum_{n=0}^{N / 4-1} \cos \left[\frac{2 \pi}{N}(2 n+1) l\right] \sum_{m=0}^{M / 2-1} u(m, n)\left\{\cos \left[\frac{\pi}{2 M}(2 m+1)(4 k+1)\right]\right. \\
& \left.-\cos \left[\frac{\pi}{2 M}(2 m+1)(4 k-1)\right]\right\} .
\end{aligned}
$$


By using (8), we have

$$
D(k, l)=\sum_{m=0}^{M / 4-1} \sum_{n=0}^{N / 4-1} v_{u}(m, n) \sin \left[\frac{2 \pi}{M}(2 m+1) k\right] \cos \left[\frac{2 \pi}{N}(2 n+1) l\right],
$$

where

$$
v_{u}(m, n)=-u(m, n) \sin \left[\frac{\pi}{2 M}(2 m+1)\right]+u(M / 2-1-m, n) \cos \left[\frac{\pi}{2 M}(2 m+1)\right] .
$$

Equation (33) can be rewritten as

$$
\begin{gathered}
D\left(\frac{M}{4}-k, l\right)=\sum_{m=0}^{M / 4-1} \sum_{n=0}^{N / 4-1}(-1)^{m} v_{u}(m, n) \cos \left[\frac{2 \pi}{M}(2 m+1) k\right] \cos \left[\frac{2 \pi}{N}(2 n+1) l\right], \\
k \in[0, M / 4-1], l \in[0, N / 4-1] .
\end{gathered}
$$

B. Computation of $C^{\prime}(k, l)$ and $D^{\prime}(k, l)$.

1) Computation of $C_{1}^{\prime}(k, l)$ and $C_{2}^{\prime}(k, l)$.

By proceeding in a similar way as for $C_{1}(k, l)$ and $C_{2}(k, l)$, we obtain

$$
\begin{aligned}
& C_{1}^{\prime}(k, l)=\frac{1}{2} \sum_{n=0}^{N / 4-1} \sin \left[\frac{2 \pi}{N}(2 n+1) l\right] \sum_{m=0}^{M / 2-1} v(m, n) \cos \left[\frac{\pi}{2 M}(2 m+1)(4 k+1)\right], \\
& C_{2}^{\prime}(k, l)=\frac{1}{2} \sum_{n=0}^{N / 4-1} \sin \left[\frac{2 \pi}{N}(2 n+1) l\right] \sum_{m=0}^{M / 2-1} v(m, n) \cos \left[\frac{\pi}{2 M}(2 m+1)(4 k-1)\right],
\end{aligned}
$$

where

$$
v(m, n)=-w(m, n) \sin \left[\frac{\pi}{2 N}(2 n+1)\right]+w(m, N / 2-1-n) \cos \left[\frac{\pi}{2 N}(2 n+1)\right] .
$$

2) Computation of $C^{\prime}(k, l)$ and $D^{\prime}(k, l)$.

By proceeding in a similar way as for $C(k, l)$ and $D(k, l)$, we have

$$
\begin{aligned}
C^{\prime}(k, l) & =\sum_{m=0}^{M / 4-1} \sum_{n=0}^{N / 4-1} u_{v}(m, n) \cos \left[\frac{2 \pi}{M}(2 m+1) k\right] \sin \left[\frac{2 \pi}{N}(2 n+1) l\right], \\
D^{\prime}(k, l) & =\sum_{m=0}^{M / 4-1} \sum_{n=0}^{N / 4-1} v_{v}(m, n) \sin \left[\frac{2 \pi}{M}(2 m+1) k\right] \sin \left[\frac{2 \pi}{N}(2 n+1) l\right],
\end{aligned}
$$

where 


$$
\begin{aligned}
& u_{v}(m, n)=v(m, n) \cos \left[\frac{\pi}{2 M}(2 m+1)\right]+v(M / 2-1-m, n) \sin \left[\frac{\pi}{2 M}(2 m+1)\right] \\
& v_{v}(m, n)=-v(m, n) \sin \left[\frac{\pi}{2 M}(2 m+1)\right]+v(M / 2-1-m, n) \cos \left[\frac{\pi}{2 M}(2 m+1)\right] .
\end{aligned}
$$

Equations (39) and (40) can also be rewritten as

$$
\begin{gathered}
C^{\prime}\left(k, \frac{N}{4}-l\right)=\sum_{m=0}^{M / 4-1} \sum_{n=0}^{N / 4-1}(-1)^{n} u_{v}(m, n) \cos \left[\frac{2 \pi}{M}(2 m+1) k\right] \cos \left[\frac{2 \pi}{N}(2 n+1) l\right] \\
k \in[0, M / 4-1], l \in[0, N / 4-1] . \\
D^{\prime}\left(\frac{M}{4}-k, \frac{N}{4}-l\right)=\sum_{m=0}^{M / 4-1} \sum_{n=0}^{N / 4-1}(-1)^{m+n} v_{v}(m, n) \cos \left[\frac{2 \pi}{M}(2 m+1) k\right] \cos \left[\frac{2 \pi}{N}(2 n+1) l\right], \\
k \in[0, M / 4-1], l \in[0, N / 4-1] .
\end{gathered}
$$

The final outputs of (11) can be obtained by

$$
\begin{aligned}
& X(2 k, 2 l)=C(k, l)+D(k, l)+C^{\prime}(k, l)+D^{\prime}(k, l), \quad k \in[0, M / 4-1], l \in[0, N / 4-1] \\
& X(2 k, 2 l-1)=C(k, l)+D(k, l)-C^{\prime}(k, l)-D^{\prime}(k, l), \quad k \in[0, M / 4-1], l \in[1, N / 4] \\
& X(2 k-1,2 l)=C(k, l)-D(k, l)+C^{\prime}(k, l)-D^{\prime}(k, l), \quad k \in[1, M / 4], l \in[0, N / 4-1] \\
& X(2 k-1,2 l-1)=C(k, l)-D(k, l)-C^{\prime}(k, l)+D^{\prime}(k, l), \quad k \in[1, M / 4], l \in[1, N / 4] .
\end{aligned}
$$

For some special values of $k$ and $l$, equation (45) can be further simplified as

$$
\begin{array}{ll}
X(2 k, 0)=C(k, 0)+D(k, 0), & \\
X(2 k-1,0)=C(k, 0)-D(k, 0), & k \in[0, M / 4] \\
X(2 k, N / 2-1)=-\left[C^{\prime}(k, N / 4)+D^{\prime}(k, N / 4)\right], & \\
X(2 k-1, N / 2-1)=-\left[C^{\prime}(k, N / 4)-D^{\prime}(k, N / 4)\right], & \\
X(0,2 l)=C(0, l)+C^{\prime}(0, l), & \\
X(0,2 l-1)=C(0, l)-C^{\prime}(0, l), & l \in[0, N / 4] \\
X(M / 2-1,2 l)=-\left[D(M / 4, l)+D^{\prime}(M / 4, l)\right], & \\
X(M / 2-1,2 l-1)=-\left[D(M / 4, l)-D^{\prime}(M / 4, l)\right], &
\end{array}
$$

and

$$
\begin{aligned}
& X(0,0)=C(0,0), \\
& X(0, N / 2-1)=-C^{\prime}(0, N / 4), \\
& X(M / 2-1,0)=-D(M / 4,0), \\
& X(M / 2-1, N / 2-1)=D^{\prime}(M / 4, N / 4) .
\end{aligned}
$$




\section{Computational complexity and comparison analysis}

In this section, we analyze the computational complexity of our proposed 2-D MDCT/IMDCT algorithm and compare it with the traditional row-column method. Assuming that a butterfly computation is implemented with 3 multiplications and 3 additions, then the decomposition costs are given as follows

1) $3 M N / 4$ additions for $w(m, n)$ in (13).

2) $3 M N / 8$ multiplications and $3 M N / 8$ additions for (28) and (38).

3) $3 M N / 8$ multiplications and $3 M N / 8$ additions for (31), (34), (41), and (42).

4) $M N / 2-M-N$ additions for (45)-(48).

In summary, the computational complexity of the proposed 2-D MDCT algorithm is given by

$$
\begin{aligned}
& M_{M \times N}^{\mathrm{MDCT}}=4 M_{(M / 4) \times(N / 4)}^{\mathrm{DCT}}+3 M N / 4, \\
& A_{M \times N}^{\mathrm{MDCT}}=4 A_{(M / 4) \times(N / 4)}^{\mathrm{DCT}}+2 M N-M-N .
\end{aligned}
$$

For the computation of 2-D IMDCT, the manipulation in (15) is just a process of data shift, $3 M N / 4$ additions can thus be saved. Fig. 1 shows the block diagram of the proposed 2-D MDCT algorithm for the case of $M=N=8$.

The time-recursive algorithm presented in [47] belonging to the recursive algorithm, is not efficient in terms of arithmetic complexity, but its regressive structure provides an efficient scheme for the parallel VLSI implementation [9]. For this reason, we compare only our algorithms with the traditional row-column method whose computational complexity is given by

$$
\begin{aligned}
& M_{M \times N}^{\mathrm{MDCT}}=M M_{N}^{\mathrm{MDCT}}+N M_{M}^{\mathrm{MDCT}}, \\
& A_{M \times N}^{\mathrm{MDCT}}=M A_{N}^{\mathrm{MDCT}}+N A_{M}^{\mathrm{MDCT}} .
\end{aligned}
$$

Note that the above equations are also valid for the 2-D IMDCT. 
In the following, we only give the comparison results of 2-D MDCT algorithm, since the results of 2-D IMDCT algorithm are only different in additions. We first consider the case where the data sequence length satisfies $M=N=2^{m}, m \geq 4$. For the proposed algorithm, we convert $M \times N$ MDCT into four $(M / 4) \times(N / 4)$ DCTs, which are then computed by the fast 2-D DCT algorithms presented in [28]-[30] or [35]. For the row-column method, we combine Lee's algorithm [4] or Cheng and Hsu's algorithm [5] with Kok's algorithm [19] for the fast computation of 1-D MDCT. The comparison result is listed in table I. Note that for $8 \times 8$-point MDCT, we used the initial values $M_{2 \times 2}^{\mathrm{DCT}}=2$ and $A_{2 \times 2}^{\mathrm{DCT}}=8[32,34]$ in table I. Then, for the case $M=N$, both $M$ and $N$ being multiple of 4 , but not power of two, we combine our 2-D MDCT algorithm with Tatsaki's algorithm [23], and compare it with row-column method, which uses the algorithm presented in [4] or [5] and Bi's algorithm [20] for the efficient computation of 1-D MDCT. The comparison result is shown in table II. For some image compression applications (e.g., [48]), it may require adaptive block sizes in different dimensions, so, we also consider the case where $M \neq N$. For the case $M=2^{m}, N=2^{n}, m \geq 3, n$ $\geq 4$, we combine our 2-D MDCT algorithm with Zeng et al's algorithm [26]. For the case $M=p \cdot 2^{m}, N=q \cdot 2^{n}, m \geq 5, n \geq 3$, we combine our 2-D MDCT algorithm with Bi et al's algorithm [37]. For the row-column method, we still use the algorithm presented in [4] or [5] and Bi's algorithm [20] for the efficient computation of 1-D MDCT. The comparison results are shown in table III and table IV, respectively. It can be observed from these tables that the proposed 2-D MDCT algorithm significantly reduces the number of arithmetic operations in both multiplications and additions compared to the row-column method. Since the block sizes $8 \times 8$ - and $16 \times 16$-point DCT are commonly used in image compression, we also consider the 
computational complexity of the $8 \times 8$ - and $16 \times 16$-point MDCT. It can be easily seen from table I that our algorithm needs only 56 (or 256) multiplications and 144 (or 776) additions for $8 \times 8$ (or $16 \times 16$ )-point DCT. However, if we use the row-column method, 128 (or 640 ) multiplications and 256 (or 1408) additions are required.

\section{Conclusions}

A fast algorithm for the computation of 2-D MDCT/IMDCT is presented. It is an extended version of an 1-D MDCT/IMDCT algorithm recently introduced by Cho et al. The algorithm reduces significantly the number of arithmetic operations compared to the row-column method. Therefore, it could find its application in multi-signal and image processing tasks.

Acknowledgement: This work was supported by National Basic Research Program of China under grant $\mathrm{N}^{0}$ 2003CB716102, Program for Changjiang Scholars and Innovative Research Team in University, and Program for New Century Excellent Talents in University under grant $\mathrm{N}^{0}$ NCET-04-0477. It has been carried out in the frame of the CRIBs, a joint international laboratory associating Southeast University, the University of Rennes 1 and INSERM, with a grant provided by the French Consulate in Shanghai. 


\section{Reference}

[1]J.P. Princen, A.W. Johnson, A.B. Bradley, Subband/Transform coding using filter bank designs based on time domain aliasing cancellation, in: Proc. IEEE ICASSP, Dallas, TX, April 1987, pp. 2161-2164.

[2]H.S. Malvar, Signal processing with lapped transforms, Artech House, Norwood, MA, 1992.

[3] V. Britanak, K.R. Rao, An efficient implementation of the forward and inverse MDCT in MPEG audio coding, IEEE Signal Process. Lett. 8(2) (February 2001) 48-51.

[4]S.W. Lee, Improved algorithm for efficient computation of the forward and backward MDCT in MPEG audio coder, IEEE Trans. Circuits Syst. II-Analog Digital Signal Process. 48(10) (October 2001) 990-994.

[5]M.H. Cheng, Y.H. Hsu, Fast IMDCT and MDCT algorithms - a matrix approach, IEEE Trans. Signal Process. 51 (1) (January 2003) 221-229.

[6]T.K. Truong, P.D. Chen, T.C. Cheng, Fast algorithm for computing the forward and inverse MDCT in MPEG audio coding, Signal Process. 86(5) (May 2006) 1055-1060.

[7]Y.K. Cho, T.H. Song, H.S. Kim, An optimized algorithm for computing the modified discrete cosine transform and its inverse transform, in: Proc. IEEE TENCON, A 21-24 November 2004, pp. 626-628.

[8]V. Britanak, K.R. Rao, A new fast algorithm for the unified forward and inverse MDCT/MDST computation, Signal Process. 82(3) (March 2002) 433-459.

[9]V. Britanak, An efficient computing of oddly stacked MDCT/MDST via evenly stacked MDCT/MDST and vice versa, Signal Process. 85(7) (July 2005) 1353-1374.

[10]H.S. Malvar, Lapped transforms for efficient transform/subband coding, IEEE Trans. Acoust., Speech, Signal Process. 38(6) (June 1990) 969-978. 
[11]H.S. Hou, S.B. Danahy, D.L. Glackin, The modulated lapped transform: avoiding artifacts in compressed remote sensing imagery, Acta Astronautica. 40(2-8) (January-April 1997) 429-435.

[12]O. Lashko, Modulated lapped transform: application in image coding and effective algorithm of its realization, in: Proc. TCSET, Slavsko, Ukraine, February 2002, pp. 243-244.

[13]T. Aach, D. Kunz, A lapped directional transform for spectral image analysis and its application to restoration and enhancement, Signal Process. 80(11) (November 2000) $2347-2364$.

[14]N.C. Tungala, A. Noore, Elimination of visual artifacts in digital image watermarking, in: Proc. $35^{\text {th }}$ Southeastern Symposium-System Theory, 16-18 March 2003, pp. 64-68.

[15]B.G. Lee, A new algorithm to compute the discrete cosine transform, IEEE Trans. Acoust., Speech, Signal Process. ASSP-32(6) (December 1984) 1243-1245.

[16]H.S. Hou, A fast recursive algorithm for computing the discrete cosine transform, IEEE Trans. Acoust. Speech Signal Process. ASSP-35(10) (October 1987) 1455-1461.

[17]C. Loeffler, A. Ligtenberg, G. S. Moschytz, Practical fast 1-D DCT algorithms with 11 multiplications, in: Proc. IEEE ICASSP, 2 February 1989, pp. 988-991.

[18]Y.H Chan, W.C. Siu, Mixed-radix discrete cosine transform, IEEE Trans. Signal Process. 41(11) (November 1993) 3157-3161.

[19]C.W. Kok, Fast algorithm for computing discrete cosine transform, IEEE Trans. Signal Process. 45(3) (March 1997) 757-760.

[20]G. Bi, L.W. Yu, DCT algorithms for composite sequence lengths, IEEE Trans. Signal Process. 46(3) (March 1998) 554-562.

[21]J. Makhoul, A fast cosine transform in one and two dimensions, IEEE Trans. Acoust. 
Speech Signal Process. ASSP-28(1) (February 1980) 27-34.

[22]N. Ta, Y. Attikiouzel, G. Crebbin, An efficient algorithm for computing two-dimensional discrete cosine transforms, in: IEEE ISCAS, 1(11-14) June 1991, pp. 396-399.

[23]A. Tatsaki, C. Dre, T. Stouraitis, C. Goutis, Prime-factor DCT algorithms, IEEE Trans. Signal Process. 43 (3) (March 1995) 772-776.

[24]P. Duhamel, C. Guillemot, Polynomial transform computation of the 2-D DCT, in: Proc. ICASSP, 3 April 1990, pp. 1515-1518.

[25]J. Prado, P. Duhamel, A polynomial-transform based computation of the 2-D DCT with minimum multiplicative complexity, in: ICASSP96, Atlanta, GA, May 1996, pp. 1347-1350.

[26]Y. Zeng, G. Bi, A.R. Leyman, New polynomial transform algorithm for multidimensional DCT, IEEE Trans. Signal Process. 48 (10) (October 2000) 2814-2821.

[27]N.I. Cho, S. U. Lee, A fast $4 \times 4$ DCT algorithm for the recursive 2-D DCT, IEEE Trans. Signal Process. 40(9) (September 1992) 2166-2173.

[28]N.I. Cho, S.U. Lee, Fast algorithm and implementation of 2-D discrete cosine transform, IEEE Trans. Circuits Syst. 38(3) (March 1991) 297-305.

[29]Y.M. Huang, J.L. Wu, A refined fast 2-D discrete cosine transform algorithm, IEEE Trans. Signal Process. 47(3) (March 1999) 904-907.

[30]Z. S. Wang, Z. Y. He, C. R. Zou, J. D. Z. Chen, A generalized fast algorithm for $n$-D discrete cosine transform and its application to motion picture coding, IEEE Trans. Circuits Syst. II—Analog Digital Signal Process. 46(5) (May 1999) 617-627.

[31]P.Z. Lee, G.S. Liu, An efficient algorithm for the 2-D discrete cosine transform, Signal Process. 55(2) (December 1996) 221-239. 
[32]F. A. Kamangar, K.R. Rao, Fast Algorithms for the 2-D Discrete Cosine Transform, IEEE Trans. Comput. C-31(9) (September 1982) 899-906.

[33]S. C. Chan, K. L. Ho, A new two-dimensional fast cosine transform algorithm, IEEE Trans. Signal Process. 39(2) (February 1991) 481-485.

[34]P.Z. Lee, F.Y. Huang, Restructured recursive DCT and DST algorithms, IEEE Trans. Signal Process. 42(7) (July 1994) 1600-1609.

[35]W.H. Fang, N.C. Hu, S.K. Shih, Recursive fast computation of the two-dimensional discrete cosine transform, IEE Proc., -Vis. Image Signal Process. 146(1) (February 1999) 25-33.

[36]V. Britanak, K.R. Rao, Two-dimensional DCT/DST universal computational structure for $2^{m} \times 2^{n}$ block sizes. IEEE Trans. Signal. Process. 48(11) (November 2000) 3250-3255.

[37]G. Bi, G. Li, K.K. Ma, T.C. Tan, On the computation of two-dimensional DCT, IEEE Trans. Signal Process. 48 (4) (April 2000) 1171-1183.

[38]J. Kwak, J. You, One- and two-dimensional constant geometry fast cosine transform algorithms and architectures, IEEE Trans. Signal Process. 47 (7) (July 1999) 2023-2034.

[39]J. Takala, D. Akopian, J. Astola, J. Saarinen, Constant geometry algorithm for discrete cosine transform, IEEE Trans. Signal Process. 48(6) (June 2000) 1840-1843.

[40]C. H. Chen, B. D. Liu, J. F. Yang, Direct recursive structures for computing radix-r two-dimensional DCT/IDCT/DST/IDST, IEEE Trans. Circuits Syst. I—Regular Papers. 51(10) (October 2004) 2017-2030.

[41]E. Feig, S. Winograd, On the multiplicative complexity of discrete cosine transforms, IEEE Trans. Info. Theory. 38(4) (July 1992) 1387-1391. 
[42]X.J. Chen, Q.H. Dai, C.W. Li, A fast algorithm for computing multidimensional DCT on certain small sizes, IEEE Trans. Signal. Process. 51(1) (January 2003) 213-220.

[43]A.M. Grigoryan, S.S. Agaian, Shifted Fourier transform-based tensor algorithms for the 2-D DCT, IEEE Trans. Signal Process. 49(9) (September 2001) 2113-2126.

[44]K.R. Rao, P. Yip, Discrete cosine transform: algorithms, advantages, applications, Academic Press, New York, 1990.

[45]H.R. Wu, Z.H. Man, Comments on "Fast algorithms and implementation of 2-D discrete cosine transform", IEEE Trans. Circuits Syst. Video Tech. 8(2) (April 1998) 128-129.

[46]W. Pan, A fast 2-D DCT algorithm via distributed arithmetic optimization, in: ICIP 2000, Vancouver, BC, 3 September 2000, pp. 114-117.

[47]E. Frantzeskakis, H.C. Karathanasis, On computing the 2-D modulated lapped transform in real-time [and VLSI implementation], in: Workshop. IEEE VLSI Signal Process., VI October 1993, pp. 361-369.

[48]J. Bracamonte, M. Ansorge, F. Pellandini, Adaptive block-size transform coding for image compression, in: Proc. IEEE ICASSP, 4 (21-24) April 1997, pp. 2721-2724. 


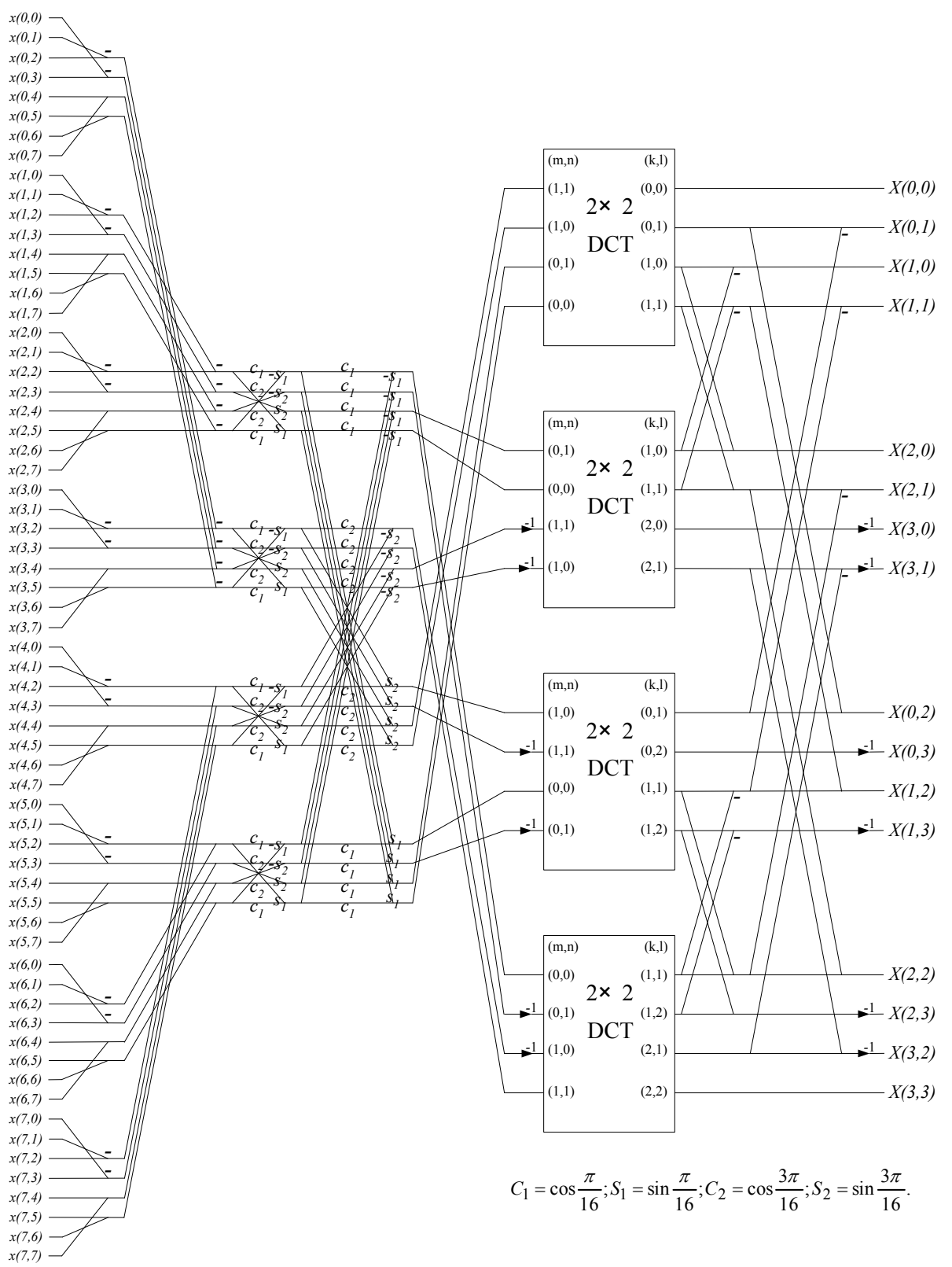

Fig. 1. The block diagram of the $8 \times 8$-point MDCT. 
Table I Required number of arithmetic operations for 2-D MDCT with block size $M \times N$, where $M=N=2^{m}, m \geq 3$.

\begin{tabular}{|c|c|c|c|c|c|c|c|}
\hline \multirow{2}{*}{$\begin{array}{c}\text { Block } \\
\text { Size }\end{array}$} & \multicolumn{3}{|c|}{ Proposed algorithm } & \multicolumn{3}{c|}{ Row-column method ([4] or [5]) } & \multirow{2}{*}{ save } \\
\cline { 2 - 7 } $8 \times 8$ & 56 & 144 & 200 & 128 & 256 & 384 & $48 \%$ \\
\hline $16 \times 16$ & 256 & 776 & 1032 & 640 & 1408 & 2048 & $50 \%$ \\
\hline $32 \times 32$ & 1152 & 3848 & 5000 & 3072 & 7168 & 10240 & $51 \%$ \\
\hline $64 \times 64$ & 5120 & 18184 & 23304 & 14336 & 34816 & 49152 & $53 \%$ \\
\hline
\end{tabular}

Table II Required number of arithmetic operations for 2-D MDCT with block size $M \times N$, where $M=N \neq 2^{m}$.

\begin{tabular}{|c|c|c|c|c|c|c|c|}
\hline \multirow{2}{*}{$\begin{array}{c}\text { Block } \\
\text { Size }\end{array}$} & \multicolumn{3}{|c|}{ Proposed algorithm } & \multicolumn{3}{c|}{ Row-column method ([4] or [5]) } & \multirow{2}{*}{ save } \\
\cline { 2 - 7 } & Mul & Add & Total & Mul & Add & Total & \\
\hline $24 \times 24$ & 568 & 1848 & 2416 & 1248 & 3456 & 4704 & $49 \%$ \\
\hline $40 \times 40$ & 1784 & 5752 & 7536 & 4480 & 11840 & 16320 & $54 \%$ \\
\hline $48 \times 48$ & 2560 & 9000 & 11560 & 6144 & 17280 & 23424 & $51 \%$ \\
\hline $56 \times 56$ & 3768 & 12728 & 16496 & 14336 & 25984 & 40320 & $59 \%$ \\
\hline
\end{tabular}

Table III Required number of arithmetic operations for 2-D MDCT with block size $M \times N$, where $M=2^{m}, N=2^{n}, m \geq 3, n \geq 4$.

\begin{tabular}{|c|c|c|c|c|c|c|c|}
\hline \multirow{2}{*}{$\begin{array}{c}\text { Block } \\
\text { Size }\end{array}$} & \multicolumn{3}{|c|}{ Proposed algorithm } & \multicolumn{3}{c|}{ Row-column method ([4] or [5]) } & \multirow{2}{*}{ save } \\
\cline { 2 - 7 } & Mul & Add & Total & Mul & Add & Total & \\
\hline $8 \times 16$ & 128 & 348 & 476 & 288 & 608 & 896 & $47 \%$ \\
\hline $16 \times 32$ & 576 & 1776 & 2352 & 1408 & 3200 & 4608 & $49 \%$ \\
\hline $32 \times 64$ & 2560 & 8536 & 11096 & 6656 & 15872 & 22528 & $51 \%$ \\
\hline \multirow{2}{*}{$64 \times 128$} & 11264 & 39592 & 50856 & 30720 & 75776 & 106496 & $52 \%$ \\
\hline
\end{tabular}

Table IV Required number of arithmetic operations for 2-D MDCT with block size $M \times N$, where $M=p \cdot 2^{m}, N=q \cdot 2^{n}, p=1, q=3, m \geq 5, n \geq 3$.

\begin{tabular}{|c|c|c|c|c|c|c|c|}
\hline \multirow{2}{*}{$\begin{array}{c}\text { Block } \\
\text { Size }\end{array}$} & \multicolumn{3}{|c|}{ Proposed algorithm } & \multicolumn{3}{c|}{ Row-column method ([4] or [5]) } & \multirow{2}{*}{ save } \\
\cline { 2 - 7 } & Mul & Add & Total & Mul & Add & Total & \\
\hline $32 \times 24$ & 984 & 2616 & 3600 & 1984 & 4992 & 6976 & $48 \%$ \\
\hline $64 \times 48$ & 4704 & 12320 & 17024 & 9472 & 24576 & 34048 & $50 \%$ \\
\hline
\end{tabular}




\begin{tabular}{|c|c|c|c|c|c|c|c|}
\hline $128 \times 96$ & 21648 & 57616 & 79264 & 44032 & 116736 & 160768 & $51 \%$ \\
\hline $256 \times 192$ & 99600 & 262000 & 361600 & 200704 & 540672 & 741376 & $51 \%$ \\
\hline
\end{tabular}

